\title{
Research Paper: Minocycline Enhance the Restorative Ability of Olfactory Ensheathing Cells by the Upregulation of BDNF and GDNF Expression After Spinal Cord Injury
}

\author{
1. Department of Animal Physiology, Faculty of Biology, Kharazmi University, Tehran, Iran \\ 2. Neuroscience Research Center, Baqiyatallah University of Medical Sciences, Tehran, Iran. \\ 3. Department of Anatomy, School of Medicine, Baqiyatallah University of Medical Sciences, Tehran, Iran.
}

Soheila Pourkhodadad $^{1 *}$ (D), Shahrbanoo Oryan' ${ }^{\text {(D), Mohammad Mehdi Hadipour }}{ }^{2}$ (D), Gholamreza Kaka² ${ }^{\text {(D), Seyed Homayoon Sadraie }}$ (iC) $^{3}$

\begin{tabular}{l|l}
$\begin{array}{c}\text { use yur devic to scan } \\
\text { and read the article online }\end{array}$ & $\begin{array}{l}\text { Cittion Pourkhodadad, S., Oryan, S., Hadipour, M. M., Kaka, G. R., \& Sadraie, S. H. (2021). Minocycline Enhance the } \\
\text { Restorative Ability of Olfactory Ensheathing Cells by the Upregulation of BDNF and GDNF Expression After Spinal Cord } \\
\text { Injury. Basic and Clinical Neuroscience, 12(6), 777-788. http://dx.doi.org/10.32598/bcn.2021.1727.1 }\end{array}$ \\
dol http://dx.doi.org/10.32598/bcn.2021.1727.1
\end{tabular}

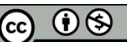

Article info:

Received: 03 Mar 2019

First Revision: 10 Apr 2020

Accepted: 27 Apr 2020

Available Online: 01 Nov 2021

Keywords:

Olfactory ensheathing cells, Minocycline, Spinal cord injury, Myelin basic protein, Brain-derived neurotrophic factor, Glial derived neurotrophic factor, Electromyography

\begin{abstract}
A B S T RAC T
Introduction: Spinal Cord Injury (SCI) is a global public health issue that results in extensive neuronal degeneration, axonal and myelin loss, and severe functional deficits. Neurotrophic factors are a potential treatment for reducing secondary damage, promoting axon growth; they are responsible for inducing myelination after injury. Olfactory Ensheathing Cells (OECs) and minocycline have promoted locomotor function after SCI. The present study investigated the neuroprotective effects of combined treatment with minocycline and OECs on spinal cord injury related to Brain-Derived Neurotrophic Factor (BDNF) and Glial Derived Neurotrophic Factor (GDNF) expressions after SCI.

Methods: Adult female rats were used to experimental SCI by weight compression method. Rats received an intraperitoneal minocycline injection $(90 \mathrm{mg} / \mathrm{kg})$ immediately after SCI and $24 \mathrm{~h}$ after injury. OECs were transplanted one week after the injury. The hindlimb function was assessed using Basso Beattie Bresnahan (BBB) locomotor rating scale and Electromyography (EMG). After 5 weeks, the spinal cord segment centered at the injury site was removed for histopathological analysis. Immunohistological and western blot assays were performed to observe the expression of NeuN, BDNF, GDNF, and Myelin Basic Protein (MBP).

Results: SCI induced the loss of locomotor function with decreased BDNF and GDNF expressions in the injury site. Minocycline+OECs increased the score of the BBB locomotor scale and increased spared tissue in the injury site. Immunohistochemical results suggested that NeuN expression significantly increased in the minocycline+OECs group than other groups. Moreover, electromyography amplitude in treated rats was increased compared to the control group. BDNF, GDNF, and MBP expressions and the number of ventral motor neurons increased further by minocycline $+\mathrm{OECs}$ in SCI rats.

Conclusion: The present study provides evidence that minocycline may facilitate recovery of locomotor function by OECs by increasing BDNF and GDNF expressions following SCI.
\end{abstract}

\section{* Corresponding Author:}




\section{Highlights}

- Combined treatment with Minocycline and OECs increased the locomotor function.

- The results showed that BDNF and GDNF expression increased by combined treatment with minocycline and OECs.

\section{Plain Language Summary}

This study examined the effect of combined treatment with minocycline and olfactory ensheathing cell on the BDNF and GDNF expression after spinal cord injury model in rat. The results showed that injection of minocycline before transplantation of OECs enhances expression of neurotrophic factors that lead to an appropriate environment for transplanted OECs and increases neuronal survival that promotes tissue sparing and functional recovery.

\section{Introduction}

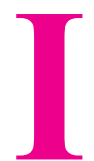

njury to the spinal cord results in extensive neuronal death, axonal degeneration, and often severe functional deficits (Devivo, 2012). Little spontaneous axonal regeneration occurs following spinal cord injury (van Niekerk, Tuszynski, Lu, \& Dulin, 2016). Several factors contribute to this lack of recovery, including glial scarring, myelin inhibition, the death of neurons and myelinating oligodendrocytes, the lack of permissive environment, and insufficient growth factor for axonal regeneration (Beattie, Li, \& Bresnahan, 2000; Huber \& Schwab, 2000). The restoration of axons depends on providing a permissive environment and enhancing neurotrophins at the injury site (Griffin \& Bradke, 2020).

Recent attempts to decrease cell death and increase axonal regeneration by enhancing the level of neurotrophic factors and blocking inhibitory molecules and inflammation have elicited axonal regrowth after SCI (Lykissas, Batistatou, Charalabopoulos, \& Beris, 2007). For instance, the beneficial effects of neurotrophic factors have been well documented on neuronal survival, axonal growth, and repair in the Central Nervous System (CNS) (Alsina, Ledda, \& Paratcha, 2012). Of these, Brain-Derived Neurotrophic Factor (BDNF) and Glial Derived Neurotrophic Factor (GDNF) have been studied extensively to find whether they have a role in promoting motor axon outgrowth of spinal cord injury (Enomoto, 2016; Ortmann \& Hellenbrand, 2018; Zhang et al., 2009). Exogenous administration of these Neurotrophic/growth factors have neuroprotective properties and limit secondary damages (Jones, Oudega, Bunge, \& Tuszynski, 2001; Rosich, Hanna, Ibrahim, Hellenbrand, \& Hanna, 2017). Previous studies reported that
BDNF promotes developmental myelination in vivo. Furthermore, it promotes CNS myelination through TrkB signaling that could be a therapeutic target for demyelinating disorders, such as stroke, Multiple Sclerosis (MS), and traumatic injury (McTigue, Horner, Stokes, \& Gage, 1998; Peckham et al., 2016; Ramos-Cejudo et al., 2015). BDNF and GDNF induce the proliferation and differentiation of oligodendrocyte progenitor cells to mature oligodandricytes. Moreover, they enhance axonal regrowth and expression of Myelin Basic Protein (MPB) in Spinal Cord Injury (SCI) (Fletcher, Jessica L, Murray, Simon \& Xiao, Junhua, 2018; Iannotti, Li, Yan, Lu, Wirthlin, \& Xu, 2003).

Minocycline, a tetracycline derivative, protects neurons against inflammation, oxidative stress, and apoptosis after spinal cord injury (Yong, Wells, Giuliani, Casha, Power, \& Metz, 2004). Besides, minocycline reduced oligodendrocyte death and improved functional recovery following SCI (Wells, Hurlbert, Fehlings, \& Yong, 2003). Recent studies indicated that minocycline is an interesting tool that reduces gliosis and promotes the production of neurotrophic factors from astrocytes, and markedly enhances the survival of injured motoneurons even when the treatment is delayed (Heydarizadi, Abbasi, Asadollahi, Rezaee, Moradipour, \& Azizi, 2019; Stirling et al., 2004). Previous studies demonstrated that minocycline could upregulate the expression of brainderived neurotrophic factors and nerve growth factors in the cerebral cortex and lumbar spinal cord; it also improve cognitive impairment via enhancing BDNF activity in the hippocampus (Zhao, Xiao, He, \& Cai, 2015).

Another mechanism to alter this environment for axonal repair can take place is using cell transplantation, such as neural stem cell, Schwann cell, and Olfactory Ensheathing Cells (OECs) (Assinck, Duncan, Hilton, Plemel, \& Tetzlaff, 2017; Heydarizadi et al., 2019). 
Indeed, OECs transplanted into the injured spinal cord express cell surfaces molecules, such as N-CAM, Ncadherin, and laminin, also produce many growth factors, including several forms of neuregulins and some neurotrophins have resulted in a remarkable degree of axonal regeneration and functional recovery (Li, Field, \& Raisman, 1998; Plant, Christensen, Oudega, \& Bunge, 2003; Ramón-Cueto, Cordero, Santos-Benito, \& Avila, 2000).

SCI is a complex complication A multifactorial molecular cascade of signals limits endogenous regenerative capacity. Accordingly, individual interventions induce small regeneration and functional recovery after SCI; a combination of neuroregenerative and neuroprotective strategies needs to overcome multiple factors to increase axonal growth (Pearse, Marcillo, Oudega, Lynch, Wood, \& Bunge, 2004). In this regard, several studies reported the importance of a combination of cell graft and pharmacological strategies after SCI (Ahuja $\&$ Fehlings, 2016). Therefore, for further investigation on this issue, the present study investigated the effect of combined treatment with minocycline and OECs on the BDNF, GDNF, MBP, and neuronal survival after SCI.

\section{Methods}

Induction of spinal cord injury models and treatment schedule

In total, 50 female Wistar rats (220-250 g) were used in biochemical experiments. The experiment protocol in the present study was approved by the animal care and ethics in Baqiyatallah University of Medical Sciences, Tehran, Iran. All animals were allocated into the following 5 groups with 10 animals in each: (1) sham group, only laminectomy; (2) control group: laminectomy+injury+saline, (3) minocycline group: lam inectomy+injury+Intraperitoneal (IP) injection of minocycline $(90 \mathrm{mg} / \mathrm{kg})$ immediately and following $24 \mathrm{~h}$ after $\mathrm{SCI}$, (4) OECs group: laminectomy+injury+OECs transplantation(450000 cells/6 $\mu$ l) 7 days after injury, and (5) minocycline+OECs group: laminectomy+injury+the injection of minocycline immediately and $24 \mathrm{~h}$ after injury $(90 \mathrm{mg} / \mathrm{kg})$ and transplantation of OECs 7 days after injury. The laminectomy was performed at the T10-T12 vertebra, and the weight $(10 \mathrm{~g})$ was dropped at the height of $2.5 \mathrm{~cm}$ to induce contusive SCI (Mukhamedshina et al., 2016). The rats were allowed free access to food and water. The bladders were emptied by manual two times per day. After 5 weeks of the appropriate treatment schedule, the rats were sacrificed with chloral hydrate, and spinal cord tissues $(2 \mathrm{~cm})$ were removed for assay or stored at $-80^{\circ} \mathrm{C}$.

\section{The preparation of OECs}

OECs were obtained from the olfactory bulb, similar to the Nash method (Nash, Borke, \& Anders, 2001). Briefly, the olfactory bulbs were dissected from meninges and blood vessels, then dissociated with mechanical trituration and incubated in $0.1 \%$ trypsin (Invitrogen) for $45 \mathrm{~min}$ at $37^{\circ} \mathrm{C}$. The cell suspension was centrifuged at $1000 \mathrm{rpm}$ for 5 minutes. After removal of trypsin, the cells resuspended in a mixture of DMEM/F12/10\% FBS and $1 \%$ penicillin /streptomycin $(\mathrm{P} / \mathrm{S}$; Invitrogen; $\mathrm{D} / \mathrm{F}-\mathrm{FBS})$; they were seeded to uncoated dishes twice, each for $24 \mathrm{~h}$ in an incubator at $37^{\circ} \mathrm{C}$ and $5 \% \mathrm{CO}_{2}$. The cells were aspirated and transferred onto $25 \mathrm{~cm} 2$ poly L-lysine (Sigma-Aldrich)-coated plastic flasks following $18 \mathrm{~h}$ of culture. The medium was changed every 2 days. Dissociated OECs were maintained in vitro for 14 days and then immunopurified using p75nerve growth factor receptor (anti-p75-NGFR, sigma). The cells were incubated with rabbit anti p75NGFR antibody (1:100; Sigma, USA) for purification. OECs purity by immunolabeling against $\mathrm{p} 75$ was at least $75 \%$.

\section{Transplantation of OECs}

The OECs were transplanted one week after injury. The laminectomy injury site was reexposed, and $6 \mu \mathrm{l}$ cell suspension $(450,000$ cells $/ 6 \mu \mathrm{L}$ for OECs) were injected by using a Hamilton syringe at a depth of $0.8 \mathrm{~mm}$ at the epicenter of the lesion and $1 \mathrm{~mm}$ rostral and caudal to the epicenter ( $2 \mu \mathrm{l}$ per injection); it reminds in place for 5 minutes after each injection (Plant et al., 2003). SCI control animals were injected with an identical volume of DMEM at the same sites. After injection, the muscle and skin were closed.

\section{Functional analysis}

The locomotor function of rats after the SCI was assessed by open field scores such as the BassoBeattieBresnahan (BBB) score (Basso, Beattie, \& Bresnahan, 1995). Two blind investigators measured scores before the injury and at $1,7,14,21,28$, and 35 days after SCI and averaged them. This scale measures hindlimb movements with a score of 0 indicating no spontaneous movement, with increasing scores for using individual joints, coordinated joint movement, coordinated limb movement, weight-bearing, and so on to a maximum score of 21 .

\section{Electromyography (EMG) evaluation}

One day before the sacrifice of the animal, rats were anesthetized, and the sciatic nerve was exposed. Electric 
stimulation was applied to the proximal site of the injured nerve. The compound muscle action potential was recorded in the gastrocnemius muscle with a needle electrode and a reference cap electrode inserted at the knee joint. The stainless steel needle used as the ground electrode was inserted into the tail skin (Chen et al., 2007).

\section{Histopathological evaluation}

The effect of combined treatment with minocycline and OECs on morphology and number of surviving neurons was determined by staining of spinal cord sections ( $\mathrm{T}$ $10 / 12,3 \mathrm{~mm}$ rostral to the epicenter) with cresyl violet and H\&E. Cross-sections at $10 \mu \mathrm{m}$ thickness were dehydrated in $100 \%$ alcohol, $95 \%$ alcohol, $70 \%$ alcohol, and distilled water. Then slices were stained in $0.1 \%$ cresyl violet and hematoxylin and eosin (Sigma-Aldrich, $\mathrm{Ph}$ 3.0) for 10 minutes at $40^{\circ} \mathrm{C}$, then washed very quickly in distilled water. Subsequently, the sections were differentiated in alcohol and xylene series and mounted. The Nisslstained cells and motor neurons in the spinal cord tissue were observed under a light microscope. Quantification was performed by counting the number of survival neurons in sections was randomly selected from each group.

\section{Immunofluorescence}

Neuronal viability was evaluated using monoclonal antibody NeuN (He et al., 2017). Sections from the lesion epicenter were collected at 35 days after SCI. The transverse sections ( $7 \mu \mathrm{m}$ thick) were deparaffinized and rehydrated to the standard protocol. Then sections were blocked for $60 \mathrm{~min}$ in 5\% Bovine Serum Albumin (BSA) (PBS containing 5\% BSA and $0.2 \%$ Triton $\mathrm{X}-100)$ and incubated with the appropriate primary antibodies, mouse monoclonal anti-NeuN ( a neuronal marker) (1:1000, Chemicon, USA) at $4{ }^{\circ} \mathrm{C}$ overnight. After washing with PBS, the secondary antibody (FITC -conjugated $\operatorname{IgG}$ ) was added, and the sections were incubated at room temperature for $1 \mathrm{~h}$. The sections were mounted, and fluorescent labeling was visualized and captured using a fluorescence microscope.

\section{Western blot analysis}

Five weeks after injury ( $\mathrm{n}=3 /$ time point), a 5 -mm spinal cord segment was dissected from each group's lesion epicenter and resuspended using RIPA lysis buffer. After centrifuging for 10 minutes $(12,000 \mathrm{rpm} /$ minutes, at $4^{\circ} \mathrm{C}$ ), for analysis by Western blot, equal amounts of each suspension sample ( $20 \mu \mathrm{g}$ protein) waere separated in $12 \%$ SDS-PAGE were transferred to PVDF membranes. Then, the blots were blocked for $1 \mathrm{~h}$ at room temperature with 5\% nonfat dry milk in Tris-Buffered Saline (TBS). After washing with TBST, the membranes were incubated with primary antibodies rabbit anti-rat antibodies against BDNF(1:200, Sigma), GDNF (1:200, Sigma), and MBP (1:200 Sigma) overnight at $4{ }^{\circ} \mathrm{C}$. The membranes were then processed with HRP-conjugated goat anti-rabbit secondary antibody (1:500; Sigma) for $1 \mathrm{~h}$ at room temperature. Immunoreactive bands were quantified using ImageJ analysis software.

All data are expressed as Mean \pm SEM and analyzed using the Graph Prism Program, Version 5. Statistical differences were determined using a one-way Analysis of Variance (ANOVA). In Fisher's Least Significant Difference (LSD) post hoc analysis, a $\mathrm{P}<0.05$, was considered to indicate a statistically significant difference.

\section{Results}

\section{Evaluations of functional recovery}

The sham, SCI, and treated animals were examined for locomotor activity on day 35 post-injury. It was observed that animals in the SCI and treated groups encountered hind limb paralysis and were unable to walk normally in the initial days after the saline or minocycline and OECs transplantation. In contrast, animals in the sham group walked normally. At 5 weeks after injury, the mean score of rats with minocycline and OECs graft were significantly higher than those for the SCI group $(\mathrm{P}<0.01, \mathrm{P}<0.05)$ (Figure 1$)$. In contrast, the $\mathrm{BBB}$ score was improved further in combined treatment with the minocycline and OECs group $(\mathrm{P}<0.001)$. There was a significant difference between OECs and minocycline + OECs groups $(\mathrm{P}<0.05)$.

\section{Valuation of EMG}

The results of the EMG tests comprised both amplitude and latency measures. The collected results indicated a statistically significant difference between control and experimental groups (OECs, minocycline, \& combined treatment with minocycline \& OECs). Moreover, results from the combined treatment group were significantly more favorable compared to the other groups $(\mathrm{P}<0.05)$ (Figure 2). There was not a significant difference between the study groups.

\section{Histopathological results}

The obtaiend data indicated that the mean cavity size was significantly less in minocycline and OECs treated groups than in the SCI group $(\mathrm{P}<0.01)$. Although the 


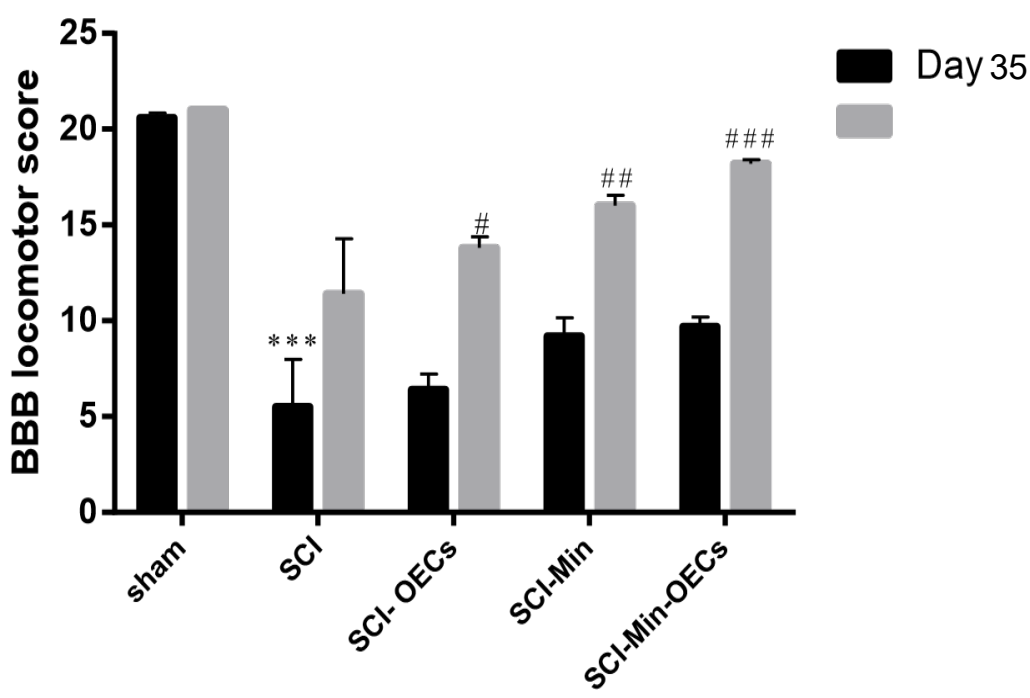

NEUR:SCIENCE

Figure 1. Locomotor function in spinal cord injury after treatment with minocycline and OECs

Rats with combined treatment with minocycline and OECs transplantation showed significantly improved locomotor behavior up to 5 weeks after SCI $(n=6)$. Data represent Mean values SEM. ${ }^{* \prime *} \mathrm{P}<0.001$ compared to sham group; $\mathrm{P}<0.05 ;{ }^{\# \# ~} \mathrm{P}<0.01$; ${ }^{\# \# \# ~} \mathrm{P}<0.001$ compared to $\mathrm{SCI}$ group.

percentage of cavitation in the OECs transplantation group showed a slight decrease compared to the minocycline group, this difference was not statistically significant $(\mathrm{P}>0.05)$. Moreover, the mean cavity area in the minocycline+OECs group was significantly reduced, comoared with SCI, OECs, and minocycline groups $(\mathrm{P}<0.001)$ (Figure 3). There was a significant difference between OECs and minocycline + OECs groups $(\mathrm{P}<0.05)$. Nissl and H\&E staining of sections showed that the number of surviving neurons and quantities of Nissl bodies in the rat spinal cord was significantly reduced in the

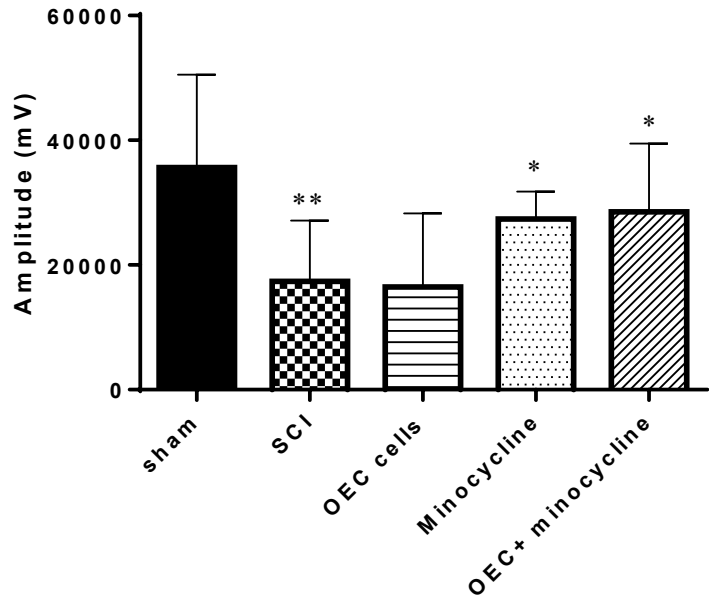

SCI group, compared with the sham group $(\mathrm{P}<0.001)$. The number of surviving neurons in the minocycline and minocycline $+\mathrm{OECs}$ groups was significantly higher than in the SCI group $(\mathrm{P}<0.01, \mathrm{P}<0.001)$. Also, the OECs group revealed a significant increase in the number of neurons compared with the SCI group $(\mathrm{P}<0.05)$ (Figure 4). There was a significant difference between OECs and minocycline+OECs groups $(\mathrm{P}<0.05)$.

Figure 2. Results of electromyography tests of amplitude and latency

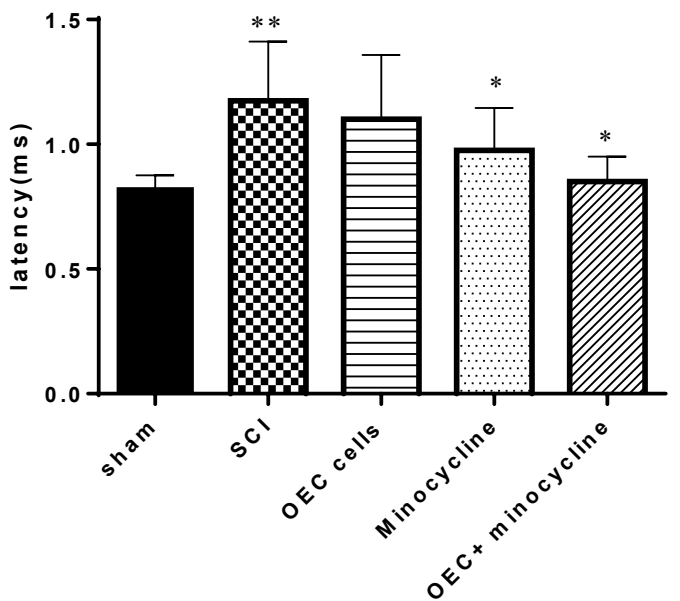

NEUR:SCIENCE

They showed there were saept statistically significant differences between the control and experimental groups and combined treatment resulted in more favorable results than the other groups $(n=6)$. Data represent Mean \pm SEM. ${ }^{*} \mathrm{P}<0.01$ vs. the sham group; ${ }^{*} \mathrm{P}<0.05$ vs. the SCI group. 


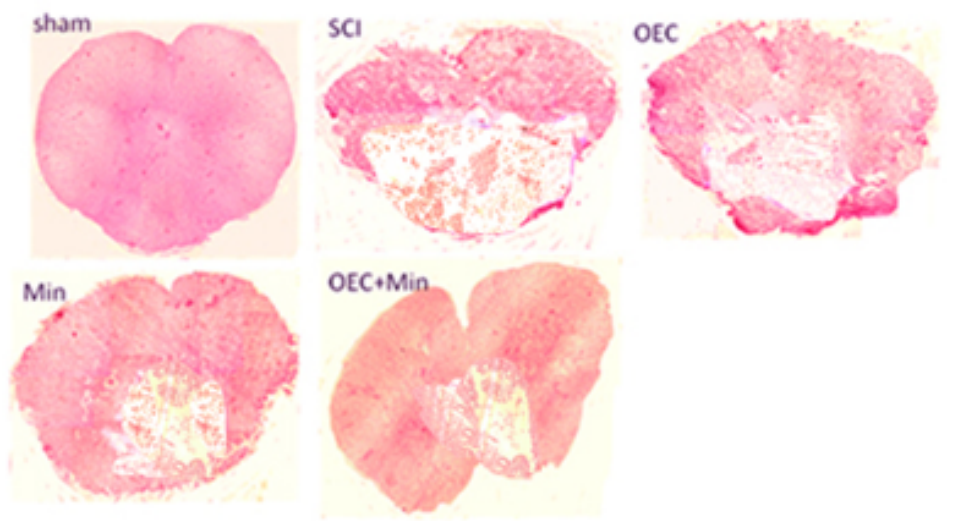

A

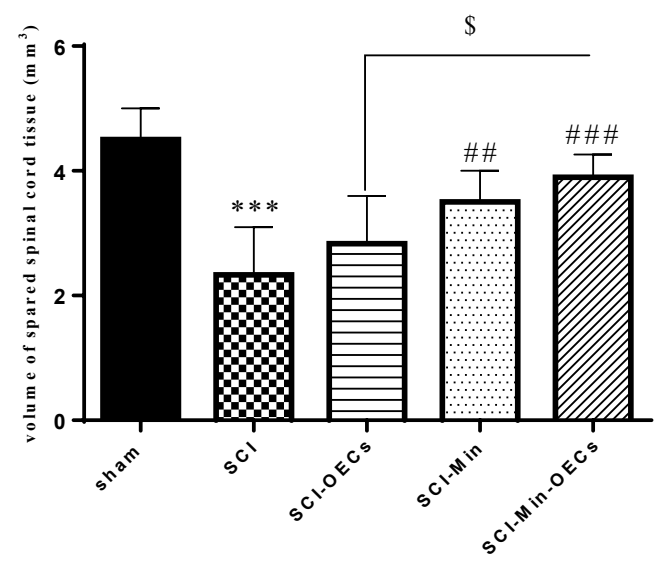

B

NEUR SCIENCE

Figure 3. Sparing of spinal cord tissue at 5 weeks after injury at the epicenter

A: Representative images of H\$E stained sections at the epicenter in the different group $(\times 10)(n=3)$; B: The volumes of spared spinal cord tissue compared between the four groups. Data represent Mean \pm SEM. ${ }^{* *} \mathrm{P}<0.001$, in SCI versus sham; ${ }^{\# \# ~} \mathrm{P}<0.01$ in minocycline versus SCI group; and ${ }^{\# \#} \mathrm{P}<0.001$ in Min+OECs versus SCI group; ${ }^{\$} \mathrm{P}<0.05$ in $\mathrm{OEC}$ s versus Min+OECs group.

\section{Expression of NeuN}

Minocycline could inhibit the inflammation and promote the mature neuron survival $(\mathrm{P}<0.01)$; when combined with OECs, the expressions of mature neuron marker NeuN were higher than other groups $(\mathrm{P}<0.001)$. Moreover, OECs alone suggested a protective effect on mature neuron survival $(\mathrm{P}<0.05)$; the lowest expression of NeuN was found in the OECs group (Figure 4). There was a significant difference between OECs and minocycline $+\mathrm{OECs}$ groups $(\mathrm{P}<0.05)$.

\section{Expression of BDNF, GDNF, and MBP}

BDNF and GDNF expression levels were significantly higher in the minocycline $(\mathrm{P}<0.01, \mathrm{P}<0.05)$ and combined treatment with minocycline and $\mathrm{OECs}$ groups $(\mathrm{P}<0.001, \mathrm{P}<0.01)$, compared with the SCI group. The expression level of BDNF was significant $(\mathrm{P}<0.05)$; however, GDNF was not significantly higher in the OECs group compared to the SCI control group. The expression levels were highest in the combined treatment with the minocycline and OECs group (Figure 5). There was a significant difference in BDNF level between OECs and minocycline+OECs groups $(\mathrm{P}<0.05)$. 

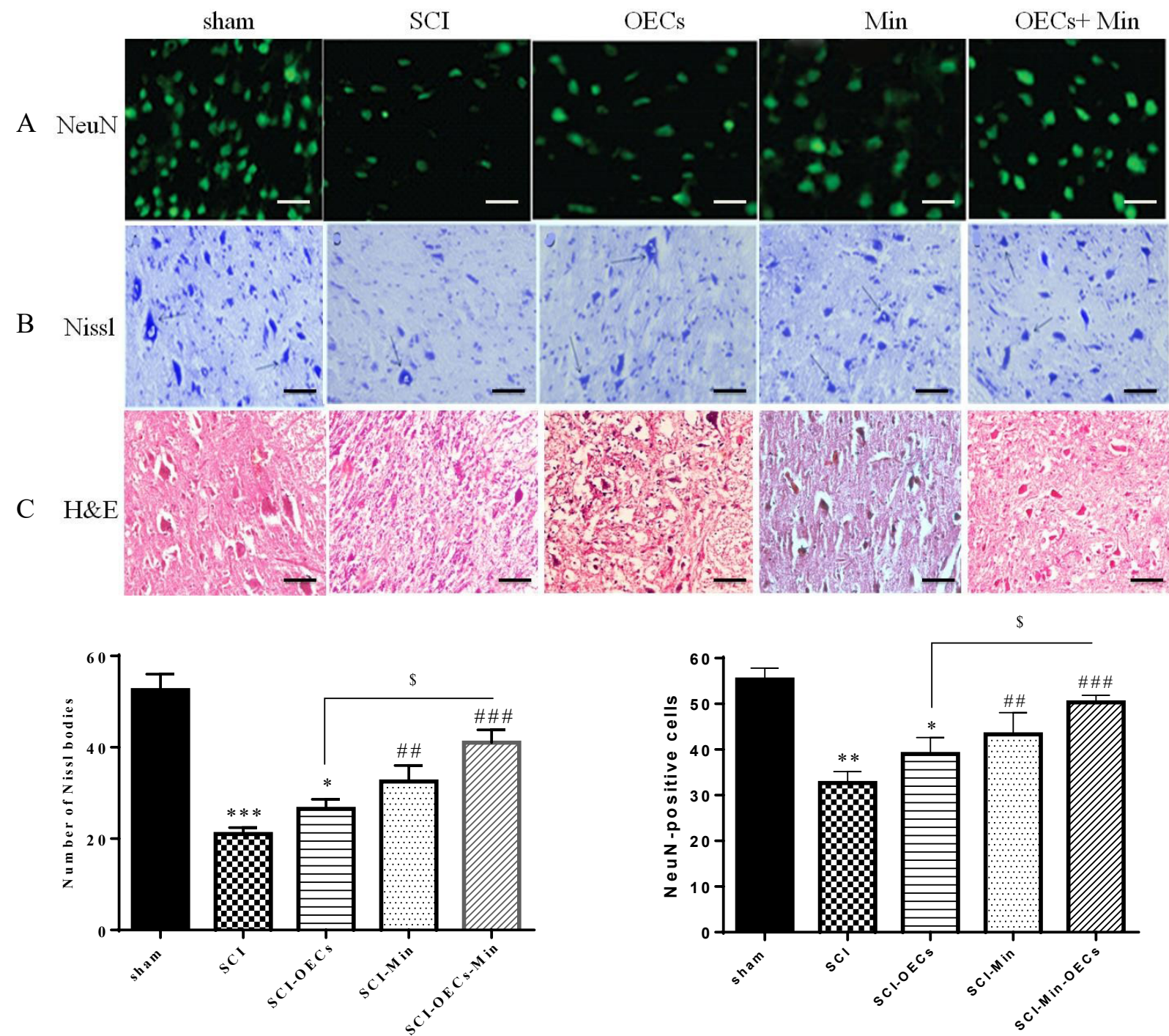

NEUR OSCIENCE

Figure 4. Combined treatment with minocycline and OECs increased motor neuronal survival

At $35 \mathrm{~d}$ after SCI, sections of the injured spinal cord were assessed via immunofluorescence staining, Nissl and H\&E staining for NeuN $(n=3)(S c a l e ~ b a r s: 50 \mu m)$. A: Immunofluorescence staining for NeuN in the different groups; B: Nissl staining results in the ventral horn of the spinal cord in the different groups at $35 \mathrm{~d}$ after SCI; C: H\&E staining results for the sections of the injured spinal cord in the experimental groups. D) The quantification analysis of the number of Nissl-stained cells; E: The quantification analysis of the number of NeuN-positive cells. Data represent Mean \pm SEM; ${ }^{* * *} \mathrm{P}<0.001$; " $\mathrm{P}<0.01$ in $\mathrm{SCI}$ versus sham; $\mathrm{P}<0.05$ in OECs versus SCI group; $\#$ " $\mathrm{P}<0.01$

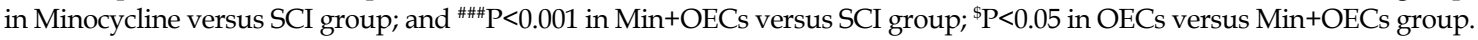

The MBP expression was compared among experimental groups to determine the density of Myelin Basic Protein (MBP) as an axonal and myelin integrity marker. There was a strong level of white matter throughout all sections of the sham group. Statistical evaluations revealed that the SCI group significantly decreased MBP expression. However, it was significantly enhanced in the minocycline and minocycline+ OECs groups, whereas the OECs group had intermediate values. Data obtained revealed that the density of MBP in the ventral horn of the spinal cord was significantly reduced in the SCI group, compared to the sham group $(\mathrm{P}<0.001)$. Moreover, the density of MBP was significantly increased in minocycline $(\mathrm{P}<0.05)$ and minocycline $+\mathrm{OECs}(\mathrm{P}<0.01)$ groups, compared with the SCI group (Figure 5). There was not a significant difference between the study groups.

\section{Discussion}

This study suggested that combined treatment with minocycline and OECs have more neuroprotective effects on the SCI. The beneficial effects of minocycline 

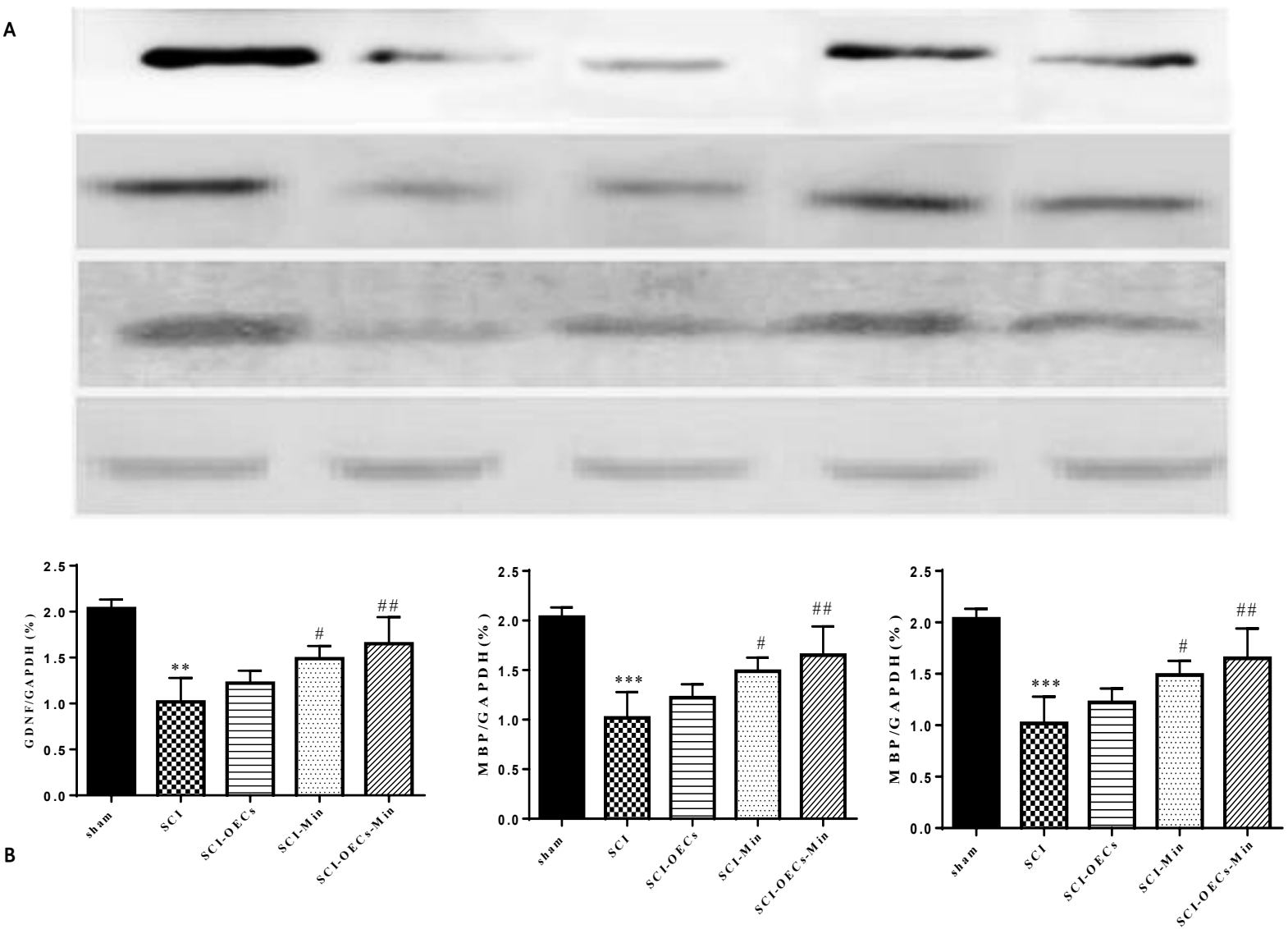

Figure 5. Effect of the combination therapy on BDNF, GDNF and MBP levels of the injured spinal cord

NEUR:SCIENCE

A: Western bloting for BDNF, GDNF and MBP in different groups (n=3); B: Quantification of protein expression of BDNF, GDNF MBP. Data are presented as Mean $\pm \mathrm{SEM}$; ${ }^{* *} \mathrm{P}<0.01$; ${ }^{* * *} \mathrm{P}<0.001$ in $\mathrm{SCI}$ versus sham group; ${ }^{*} \mathrm{P}<0.05$ in OECs versus SCI group; \#P<0.05; \#\#P<0.01;

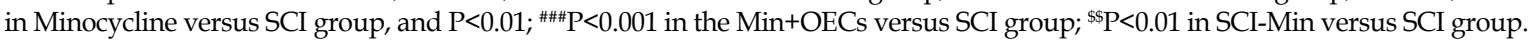

and OECs on SCI may be related to multiple aspects. For the first time, we found that minocycline injection could upregulate the expression of BDNF and GDNF in a contusion model of spinal cord injury. Our results indicated that combined treatment with OECs and minocycline into the injured spinal cord significantly improved the recovery of locomotor function, reduced spinal cord damage, and resulted in significantly more spared tissue than individual treatment.

Additionally, BBB and EMG analyses found significant improvement in locomotor function in minocycline and minocycline $+\mathrm{OECs}$ groups at 5 weeks post-injury. Combined treatment with minocycline and OECs were more effective than individual treatment. In addition, minocycline + OECs significantly increased spared white matter and myelin at the injured epicenter.

Minocycline serves as an antiinflammation agent, protects motoneurons and oligodendrocytes against inflammation, oxidative stress, and apoptosis, and promotes neu- ral regeneration (Ahmad, Zakaria, \& Almutairi, 2016; Festoff, Ameenuddin, Arnold, Wong, Santacruz, \& Citron, 2006). Furthermore, minocycline has been demonstrated to suppress astrocyte reactivation and reduce neuronal apoptosis following SCI (Festoff et al., 2006).

In this study, to further clarify the mechanism by which minocycline and OECs improved locomotor deficits, the expression of BDNF and GDNF were investigated. BDNF and GDNF are the neurotrophins that play a critical role in the development of the brain and are involved in structural remodeling, neuronal plasticity, and synaptic restructuring (Hellweg \& JockersScherübl, 1994). BDNF and GDNF play essential roles in improving locomotor function after SCI through neural protection and neural regeneration by various cellular mechanisms (Jones, Oudega, Bunge, \& Tuszynski, 2001).

In the present study, BDNF and GDNF were downregulated in the spinal cord tissue after contusive injury, whereas BDNF and GDNF were upregulated af- 
ter minocycline and OECs transplantation. Moreover, statistical analysis sugegsted that the effects of OECs transplantation alone were better than those of SCI and increased BDNF. A combination of OECs and minocycline significantly bettered the above detection indexes compared with the SCI model group at 35 days. Thus, it is speculated that minocycline induces a neuroprotective effect in contusion models of SCI through enhancing the expression of BDNF and GDNF in the spinal cord. This result was consistent with the previous reports that minocycline could up-regulate the expression of BDNF and Nerve Growth Factor (NGF) both in the cerebral cortex and lumbar spinal cord of mice with experimental autoimmune encephalomyelitis (Chen et al., 2012). Besides, minocycline increased the expression of BDNF and improved cognitive suffered from impairment of permanent bilateral occlusion (Zhao et al., 2015).

Moreover, OECs may overcome the injury site inhibition and promote neurite sprouting and outgrowth by providing both an adhesive cellular substrate and permissive soluble factors (Ramón-Cueto \& Valverde, 1995; Sonigra, Brighton, Jacoby, Hall, \& Wigley, 1999; Barnett \& Riddell, 2007) that may serve as the significant factors for promoting nerve growth, regeneration, and repair (Mills, Allchorne, Griffin, Woolf, \& Costigan, 2007; Spencer, Mellado, \& Filbin, 2008). It is reported that the therapeutic potential of olfactory ensheathing cells in spinal cord repair can enhance using neurotrophins (Wright et al., 2018). The obatined study determined that OECs increased BDNF one week after injury, reduced cell death, and induced neuroprotective effect against inflammation. This has been indicated in NeuN expression and nissl staining. Thus, it is speculated that minocycline and OECs have neuroprotective effects in contusive spinal cord injury by enhancing BDNF and GDNF in the SCI. Combined treatment induces more neuroprotective effects than individual treatment.

We used NeuN as an in vivo marker of mature neuronal survival and nissl staining following SCI to confirm these results. NeuN is an antigen used widely in research and diagnostics to identify postmitotic neurons (Gusel'Nikova \& Korzhevskiy, 2015). Moroever, we observed that the NeuN expression and number of nissl staining in the minocycline+OECs group were more significant than in the minocycline and OECs groups. In other words, minocycline enhanced neurotrophic factor levels combined with OECs promote neuronal survival and have the best protective effect on mature neurons.

Besides, a combination of minocycline with OECs transplantation promoted MBP expression and preserved white matter after contusion spinal cord injury than other groups (Figure 4). Thus, up-regulation of MBP expression may reflect the axonal repair response that leads to recovery of the spinal cord function (Ruitenberg et al., 2003). This study has shown that minocycline increases BDNF and GDNF expressions, reducing oligodendrocyte apoptosis and promoting the upregulation of MBP (Ikeda et al., 2002; Koda et al., 2002). One study outlined that minocycline injection improves functional recovery after traumatic spinal cord injury by reducing oligodendrocytes death (Yune et al., 2007). Per previous studies (Li et al., 2011), the results of MBP expression in the OECs transplantation group showed a partial increase in MBP expression and remyelination than SCI group. Several studies have reported that OECs can remyelination of axons; however, it remains unclear whether OECs are directly or indirectly responsible for this process (Franssen, de Bree, \& Verhaagen, 2007).

\section{Conclusion}

In conclusion, the injection of minocycline before OECs graft could increase neurotrophic factors such as BDNF and GDNF that result in a favorable environment for OECs graft and contribute to the ability of OECs to enhance axon regeneration after SCI.

\section{Ethical Considerations}

\section{Compliance with ethical guidelines}

All experimental protocols were in strict accordance with the guidelines for animal research, as detailed in the NIH Guidelines for the Care and Use of Laboratory Animals.

Funding

This work was supported financially by Iran National Science Foundation (INSF, Grant no.: 94803041).

Authors' contributions

All authors contributed to preparing this study equally.

\section{Conflict of interest}

The authors declared no conflict of interest.

\section{References}

Ahmad, M., Zakaria, A., \& Almutairi, K.M. (2016). Effectiveness of minocycline and FK506 alone and in combination on enhanced behavioral and biochemical recovery from spinal cord injury in rats. Pharmacology Biochemistry and Behavior, 145, 45-54 [DOI:10.1016/j.pbb.2016.04.003] [PMID] 
Ahuja, C. S., \& Fehlings, M. (2016). Concise review: Bridging the gap: Novel neuroregenerative and neuroprotective strategies in spinal cord injury. Stem Cells Translational Medicine, 5(7), 914-24. [DOI:10.5966/sctm.2015-0381] [PMID] [PMCID]

Alsina, F. C., Ledda, F., \& Paratcha, G. (2012). New insights into the control of neurotrophic growth factor receptor signaling: Implications for nervous system development and repair. Journal of Neurochemistry, 123(5), 652-61. [DOI:10.1111/ jnc.12021] [PMID]

Assinck, P., Duncan, G. J., Hilton, B. J., Plemel, J. R., \& Tetzlaff, W. (2017). Cell transplantation therapy for spinal cord injury. $\mathrm{Na}$ ture Neuroscience, 20(5), 637-47. [DOI:10.1038/nn.4541] [PMID]

Barnett, S. C., \& Riddell, J. S. (2007). Olfactory ensheathing cell transplantation as a strategy for spinal cord repair-what can it achieve? Nature Clinical Practice Neurology, 3(3), 152-61. [DOI:10.1038/ncpneuro0447] [PMID]

Basso, D. M., Beattie, M. S., \& Bresnahan, J. C. (1995). A sensitive and reliable locomotor rating scale for open field testing in rats. Journal of Neurotrauma, 12(1), 1-21. [DOI:10.1089/ neu.1995.12.1] [PMID]

Beattie, M. S., Li, Q., \& Bresnahan, J. C. (2000). Cell death and plasticity after experimental spinal cord injury. Progress in Brain Research, 128, 9-21. [DOI:10.1016/S0079-6123(00)280035] [PMID]

Chen, C. J., Ou, Y. C., Liao, S. L., Chen, W. Y., Chen, S. Y., \& $\mathrm{Wu}, \mathrm{C}$. W., et al. (2007). Transplantation of bone marrow stromal cells for peripheral nerve repair. Experimental Neurology, 204(1), 443-53. [DOI:10.1016/j.expneurol.2006.12.004] [PMID]

Chen, X., Ma, L., Jiang, Y., Chen, S., Zhu, C., \& Liu, M., et al. (2012). Minocycline up-regulates the expression of brainderived neurotrophic factor and nerve growth factor in experimental autoimmune encephalomyelitis. European Journal of Pharmacology, 686(1-3), 124-9. [DOI:10.1016/j. ejphar.2012.04.043] [PMID]

Pearse, D. D., Marcillo, A. E., Oudega, M., Lynch, M. P., Wood, P. M., \& Bunge, M. B. (2004). Transplantation of Schwann cells and olfactory ensheathing glia after spinal cord injury: Does pretreatment with methylprednisolone and interleukin-10 enhance recovery? Journal of Neurotrauma, 21(9), 1223-39. [DOI:10.1089/neu.2004.21.1223] [PMID]

Devivo, M. J. (2012). Epidemiology of traumatic spinal cord injury: Trends and future implications. Spinal Cord, 50(5), 365-72. [DOI:10.1038/sc.2011.178] [PMID]

Enomoto, M. (2016). Therapeutic effects of neurotrophic factors in experimental spinal cord injury models. Journal of Neurorestoratology, 4(1), 15-22. [DOI:10.2147/JN.S66874]

Festoff, B. W., Ameenuddin, S., Arnold, P. M., Wong, A., Santacruz, K. S., \& Citron, B. A. (2006). Minocycline neuroprotects, reduces microgliosis, and inhibits caspase protease expression early after spinal cord injury. Journal of Neurochemistry, 97(5), 1314-26. [DOI:10.1111/j.1471-4159.2006.03799.x] [PMID]

Fletcher, Jessica L, Murray, Simon S, \& Xiao, Junhua. (2018). Brainderived neurotrophic factor in central nervous system myelination: A new mechanism to promote myelin plasticity and repair. International Journal of Molecular Sciences, 19(12), 4131. [DOI:10.3390/ijms19124131] [PMID] [PMCID]

Franssen, E. H., de Bree, F. M., \& Verhaagen, J. (2007). Olfactory ensheathing glia: Their contribution to primary olfactory nervous system regeneration and their regenerative potential following transplantation into the injured spinal cord. Brain Research Reviews, 56(1), 236-58. [DOI:10.1016/j.brainresrev.2007.07.013] [PMID]

Griffin, J. M., \& Bradke, F. (2020). Therapeutic repair for spinal cord injury: Combinatory approaches to address a multifaceted problem. EMBO Molecular Medicine, 12(3), e11505. [DOI:10.15252/emmm.201911505] [PMID] [PMCID]

Guselnikova, V. V, \& Korzhevskiy, D. E. (2015). NeuN as a neuronal nuclear antigen and neuron differentiation marker. Acta Naturae, 7(2), 42-7. [DOI:10.32607/20758251-2015-7-2-42-47] [PMID] [MPCID]

He, Z., Zhou, Y., Huang, Y., Wang, Q., Zheng, B., \& Zhang, H., et al. (2017). Dl-3-n-butylphthalide improves functional recovery in rats with spinal cord injury by inhibiting endoplasmic reticulum stress-induced apoptosis. American Journal of Translational Research, 9(3), 1075-87. [PMID] [PMCID]

Hellweg, R., \& Jockers-Scherübl, M. (1994). Neurotrophic factors in memory disorders. Life Sciences, 55(25-26), 2165-9. [DOI:10.1016/0024-3205(94)00397-1] [PMID]

Heydarizadi, S., Abbasi, N., Asadollahi, KH., Rezaee, S., Moradipour, A., \& Azizi, M. (2019). Effects of transplanted olfactory ensheathing cells on functional improvement and axonal regeneration in acute and delayed spinal cord injury in rats: A comparative study. Journal of the Anatomical Society of India 68(1), 1-6. [DOI: 10.4103/JASI.JASI_21_19]

Huber, A. B., \& Schwab, M. E. (2000). Nogo-A, a potent inhibitor of neurite outgrowth and regeneration. Biological Chemistry, 381(5-6), 407-19. [DOI:10.1515/BC.2000.053] [PMID]

Iannotti, C., Li, H., Yan, P., Lu, X., Wirthlin, L., \& Xu, X. M. (2003). Glial cell line-derived neurotrophic factor-enriched bridging transplants promote propriospinal axonal regeneration and enhance myelination after spinal cord injury. Experimental Neurology, 183(2), 379-93. [DOI:10.1016/S0014-4886(03)001882] [PMID]

Ikeda, O., Murakami, M., Ino, H., Yamazaki, M., Koda, M., \& Nakayama, C., et al. (2002). Effects of brain-derived neurotrophic factor (BDNF) on compression-induced spinal cord injury: BDNF attenuates downregulation of superoxide dismutase expression and promotes up-regulation of myelin basic protein expression. Journal of Neuropathology \& Experimental Neurology, 61(2), 142-53. [DOI:10.1093/jnen/61.2.142] [PMID]

Jones, L. L., Oudega, M., Bunge, M. B., \& Tuszynski, M. H. (2001). Neurotrophic factors, cellular bridges and gene therapy for spinal cord injury. Journal of Physiology, 533(1), 83-9. [DOI:10.1111/j.1469-7793.2001.0083b.x] [PMID] [PMCID]

Koda, M., Murakami, M., Ino, H., Yoshinaga, K., Ikeda, O., \& Hashimoto, M., et al (2002). Brain-derived neurotrophic factor suppresses delayed apoptosis of oligodendrocytes after spinal cord injury in rats. Journal of Neurotrauma, 19(6), 777-85. [DOI:10.1089/08977150260139147] [PMID]

Li, Y., Bao, J., Khatibi, N. H., Chen, L., Wang, H., \& Duan, Y., et al. (2011). Olfactory ensheathing cell transplantation into spinal cord prolongs the survival of mutant sod1g93a als rats through neuroprotection and Remyelination. The Anatomical Record 294(5), 847-57. [DOI:10.1002/ar.21362] [PMID]

Li, Y., Field, P. M., \& Raisman, G. (1998). Regeneration of adult rat corticospinal axons induced by transplanted olfactory ensheath- 
ing cells. Journal of Neuroscience, 18(24), 10514-24. [DOI:10.1523/ JNEUROSCI.18-24-10514.1998] [PMID] [PMCID]

Lykissas, M. G., Batistatou, A. K, Charalabopoulos, K. A., \& Beris, A. E. (2007). The role of neurotrophins in axonal growth, guidance, and regeneration. Current Neurovascular Research, 4(2), 14351. [DOI:10.2174/156720207780637216] [PMID]

McTigue, D. M., Horner, P. J., Stokes, B. T., \& Gage, F. H. (1998) Neurotrophin 3 and brain-derived neurotrophic factor induce oligodendrocyte proliferation and myelination of regenerating axons in the contused adult rat spinal cord. Journal of Neuroscience, 18(14), 5354-65. [DOI:10.1523/JNEUROSCI.18-14-05354.1998] [PMID] [PMCID]

Mills, C. D., Allchorne, A. J., Griffin, R. S., Woolf, C. J., \& Costigan, M. (2007). GDNF selectively promotes regeneration of injury-primed sensory neurons in the lesioned spinal cord. Molecular and Cellular Neuroscience, 36(2), 185-94. [DOI:10.1016/j. mcn.2007.06.011] [PMID] [PMCID]

Mukhamedshina, Y. O., Shaymardanova, G. F., Garanina, E. E., Salafutdinov, I. I., Rizvanov, A. A., \& Islamov, R. R., et al. (2016). Adenoviral vector carrying glial cell-derived neurotrophic factor for direct gene therapy in comparison with human umbilical cord blood cell-mediated therapy of spinal cord injury in rat. Spinal Cord, 54(5), 347-59. [DOI:10.1038/sc.2015.161] [PMID]

Nash, H. H., Borke, R. C, \& Anders, J. J. (2001). New method of purification for establishing primary cultures of ensheathing cells from the adult olfactory bulb. Glia, 34(2), 81-7. [DOI:10.1002/ glia.1043] [PMID]

Ortmann, S. D., \& Hellenbrand, D. J. (2018). Glial cell line-derived neurotrophic factor as a treatment after spinal cord injury. Neural Regeneration Research, 13(10), 1733-4. [DOI:10.4103/16735374.238610] [PMID] [PMCID]

Peckham, H, Giuffrida, L, Wood, R., Gonsalvez, D., Ferner, A, \& Kilpatrick, T. J., et al. (2016). Fyn is an intermediate kinase that BDNF utilizes to promote oligodendrocyte myelination. Glia, 64(2), 255-69. [DOI:10.1002/glia.22927] [PMID]

Plant, G. W., Christensen, C. L., Oudega, M., \& Bunge, M. B. (2003). Delayed transplantation of olfactory ensheathing glia promotes sparing/regeneration of supraspinal axons in the contused adult rat spinal cord. Journal of Neurotrauma, 20(1), 1-16. [DOI:10.1089/08977150360517146] [PMID]

Ramón-Cueto, A., Cordero, M. I., Santos-Benito, F. F., \& Avila, J. (2000). Functional recovery of paraplegic rats and motor axon regeneration in their spinal cords by olfactory ensheathing glia. Neuron, 25(2), 425-35. [DOI:10.1016/S0896-6273(00)80905-8] [PMID]

Ramón-Cueto, A., \& Valverde, F. (1995). Olfactory bulb ensheathing glia: A unique cell type with axonal growth-promoting properties. Glia, 14(3), 163-73. [DOI:10.1002/glia.440140302] [PMID]

Ramos-Cejudo, J., Gutiérrez-Fernández, M., Otero-Ortega, L., Rodríguez-Frutos, B., Fuentes, B., \& Vallejo-Cremades, M. T., et al. (2015). Brain-derived neurotrophic factor administration mediated oligodendrocyte differentiation and myelin formation in subcortical ischemic stroke. Stroke, 46(1), 221-8. [DOI:10.1161/ STROKEAHA.114.006692] [PMID]

Rosich, K., Hanna, B. F., Ibrahim, R. K., Hellenbrand, D. J., \& Hanna, A. (2017). The effects of glial cell line-derived neurotrophic factor after spinal cord injury. Journal of Neurotrauma, 34(24), 3311-25. [DOI:10.1089/neu.2017.5175] [PMID]

Ruitenberg, M. J., Plant, G. W., Hamers, F. P. T., Wortel, J., Blits, B., \& Dijkhuizen, P. A., et al. (2003). Ex vivo adenoviral vector- mediated neurotrophin gene transfer to olfactory ensheathing glia: Effects on rubrospinal tract regeneration, lesion size, and functional recovery after implantation in the injured rat spinal cord. Journal of Neuroscience, 23(18), 7045-58. [DOI:10.1523/ JNEUROSCI.23-18-07045.2003] [PMID] [PMCID]

Sonigra, R. J., Brighton, P. C., Jacoby, J., Hall, S., \& Wigley, C. B. (1999). Adult rat olfactory nerve ensheathing cells are effective promoters of adult central nervous system neurite outgrowth in coculture. Glia, 25(3), 256-69. [DOI:10.1002/(sici)10981136(19990201)25:3\%3C256::aid-glia6\%3E3.0.co;2-y] [PMID]

Spencer, T. K., Mellado, W., \& Filbin, M. T. (2008). BDNF activates CaMKIV and PKA in parallel to block MAG-mediated inhibition of neurite outgrowth. Molecular and Cellular Neuroscience, 38(1), 110-16. [DOI:10.1016/j.mcn.2008.02.005] [PMID] [PMCID]

Stirling, D. P., Khodarahmi, K., Liu, J., McPhail, L. T., McBride, C, B., \& Steeves, J. D., et al. (2004). Minocycline treatment reduces delayed oligodendrocyte death, attenuates axonal dieback, and improves functional outcome after spinal cord injury. Journal of Neuroscience, 24(9), 2182-90. [DOI:10.1523/ JNEUROSCI.5275-03.2004] [PMID] [PMCID]

van Niekerk, E. A., Tuszynski, M. H., Lu, P., \& Dulin, J. N. (2016) Molecular and cellular mechanisms of axonal regeneration after spinal cord injury. Molecular \& Cellular Proteomics, 15(2), 394-408. [DOI:10.1074/mcp.R115.053751] [PMID] [PMCID]

Wells, J. E. A., Hurlbert, R. J., Fehlings, M. G., \& Yong, V. W. (2003). Neuroprotection by minocycline facilitates significant recovery from spinal cord injury in mice. Brain, 126(Pt7), 1628-37. [DOI:10.1093/brain/awg178] [PMID]

Wright, A. A., Todorovic, M., Tello-Velasquez, J., Rayfield, A. J., St John, J. A., \& Ekberg, J. A., et al. (2018). Enhancing the therapeutic potential of olfactory ensheathing cells in spinal cord repair using neurotrophins. Cell Transplantation, 27(6), 867-78. [DOI:10.1177/0963689718759472] [PMID] [PMCID]

Yong, V. W., Wells, J., Giuliani, F., Casha, S., Power, C., \& Metz, L. M. (2004). The promise of minocycline in neurology. The Lancet Neurology, 3(12), 744-51. [DOI:10.1016/S14744422(04)00937-8] [PMID]

Yune, T. Y., Lee, J. Y., Jung, G. Y., Kim, S. J., Jiang, M. H, \& Kim, Y. C., et al. (2007). Minocycline alleviates death of oligodendrocytes by inhibiting pro-nerve growth factor production in microglia after spinal cord injury. Journal of Neuroscience, 27(29), 7751-61. [DOI:10.1523/JNEUROSCI.1661-07.2007] [PMID] [PMCID]

Zhang, L., Ma, Z., Smith, G. M., Wen, X., Pressman, Y., \& Wood, P. M., et al. (2009). GDNF-enhanced axonal regeneration and myelination following spinal cord injury is mediated by primary effects on neurons. Glia, 57(11), 1178-91. [DOI:10.1002/ glia.20840] [PMID] [PMCID]

Zhao, Y., Xiao, M., He, W., \& Cai, Z. (2015). Minocycline upregulates cyclic AMP response element binding protein and brainderived neurotrophic factor in the hippocampus of cerebral ischemia rats and improves behavioral deficits. Neuropsychiatric Disease and Treatment, 11, 507-16. [DOI:10.2147/ndt.s73836] [PMID] [PMCID] 
This Page Intentionally Left Blank 